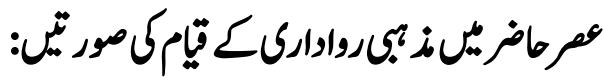

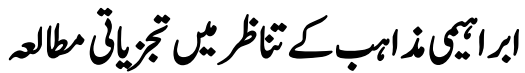

\section{Ways of Establishing Religious Pluralism in Contemporary Age: An Analytical Study of Abrahamic Religions}

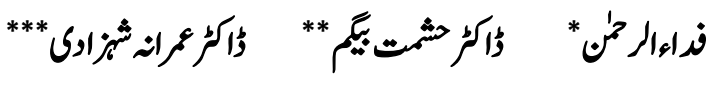

\section{فراءارحز}

ISSN (P) 2664-0031 (E) 2664-0023

DOI: https://doi.org/10.37605/fahmiislam.v4i1.218
Received: February 12,2021

Accepted: February 22, 2021

Published: June 30,2021

\begin{abstract}
Human being is endowed with intellect. The standard and domain of intellectual exposure are different and diverse. Consequently, it gives birth to diversity in opinions and perspective that eventually results in mutual disagreement. A deep assessment of the human life indicates the fact that diversity exist in almost all aspects of human life. The diversity of opinions, perspectives and narratives can be found with reference to culture and civilization, economy, entrepreneurship, modern scientific disciplines, politics and religion. The diversity is an indication of the evolution in human intellect. When the diversity is misunderstood as a negative connotation then it turns into conflict that results into incalculable and disastrous impacts on society. Pluralism plays a pivotal role in the evolution of diverse human society and multicultural civilization and a pluralistic society can tread the phases of socio-cultural development more conveniently and productively. If a society becomes an arena of religious intolerance, hatred and biasness then the fate of society becomes uncertain and bleak. It is need of the day to create awareness and pursue research studies regarding pluralism while keeping in view the golden traditions of Abrahamic religions. The present paper is an attempt to explore the fundamental principles that deal with religious tolerance and pluralism in Abrahamic religions. This paper will help to create awareness about the need and importance of tolerance and pluralism and will suggest recommendations for implementation of those principles both at state and society levels so that the culture of tolerance, pluralism, mutual understanding and human dignity are promoted in wider interest of a unified and peaceful society.
\end{abstract}

Keywords: Abrahamic Religions, Tolerance, diversity, Pluralism.

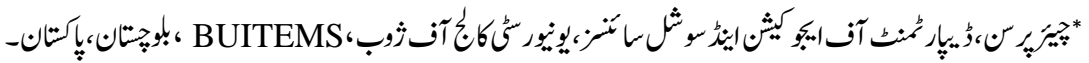

$$
\begin{aligned}
& \text { Fida.ucz.buitems@gmail.com }
\end{aligned}
$$

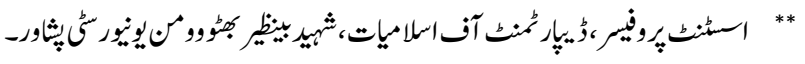

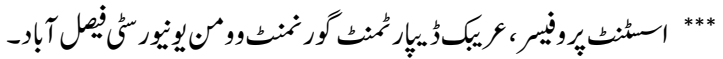




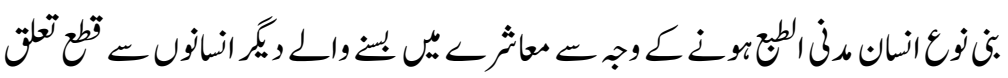

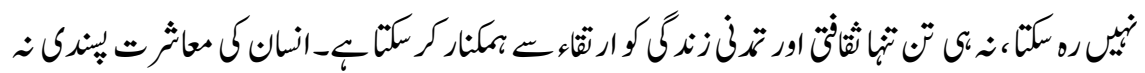

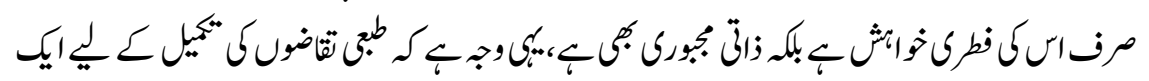

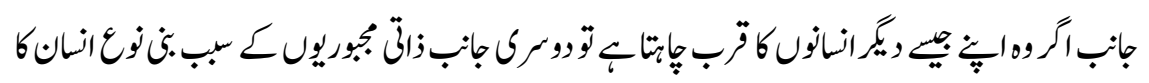

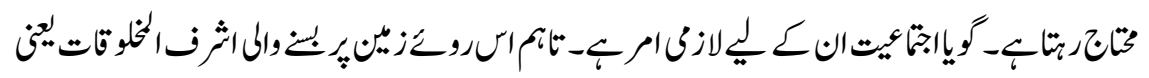

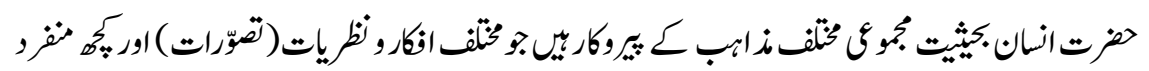

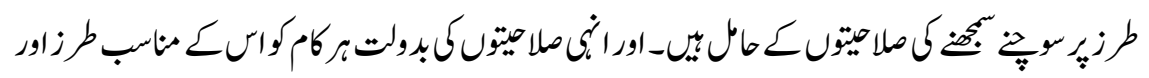

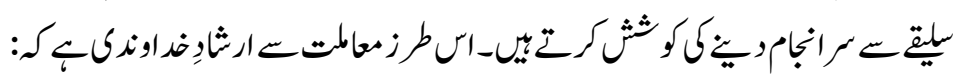

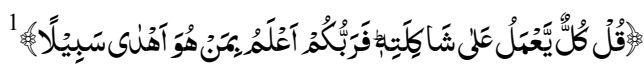

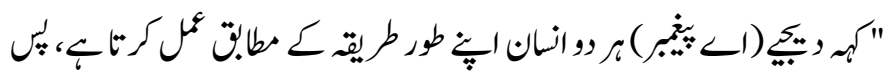

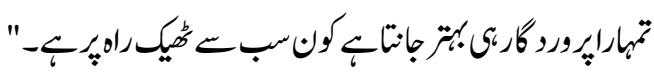

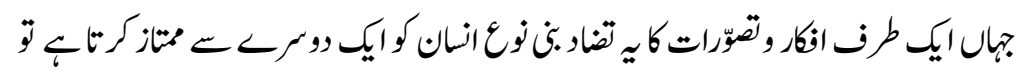

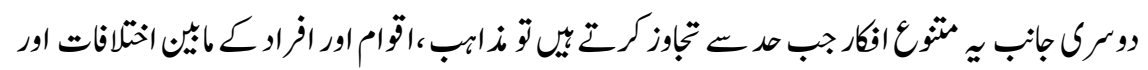

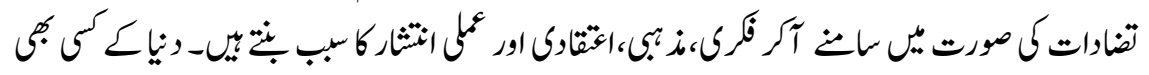

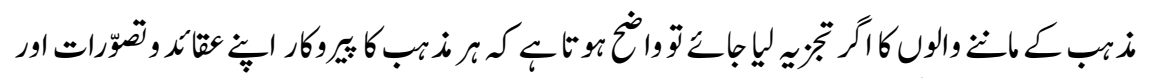

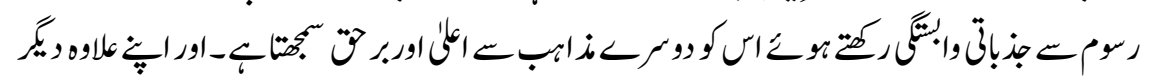

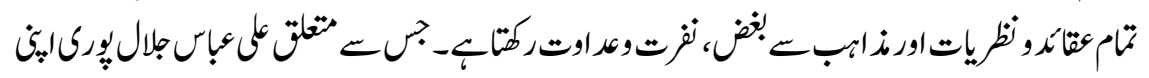

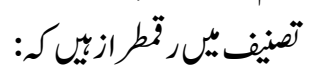

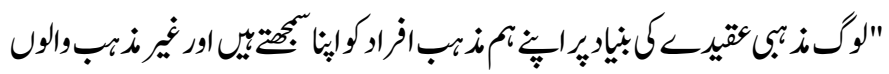

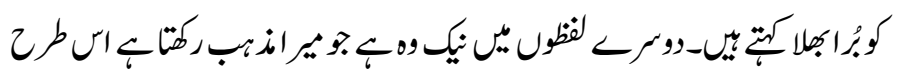

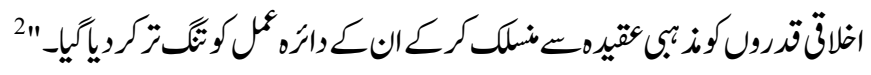

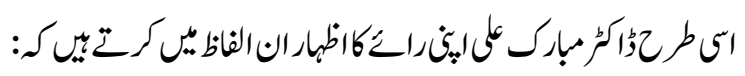

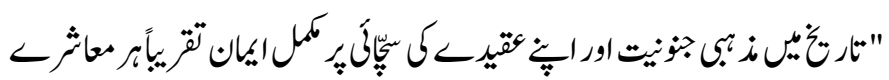

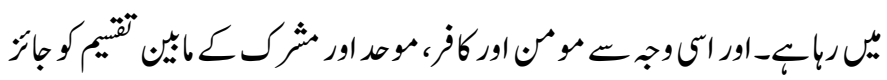




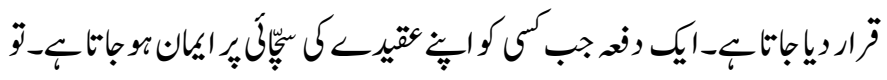

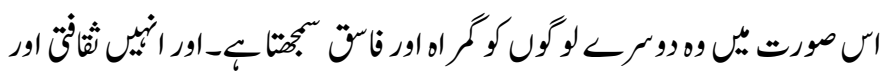

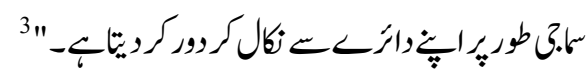

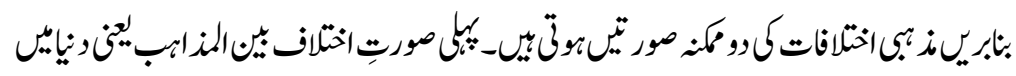

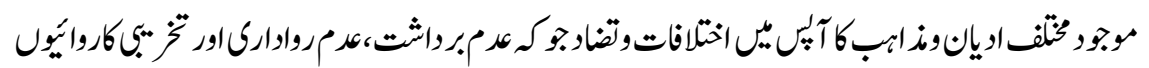

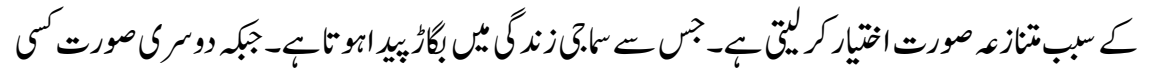

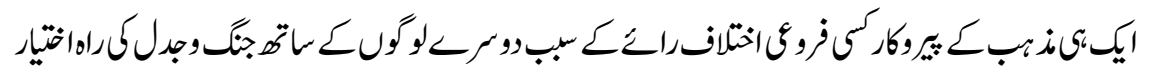

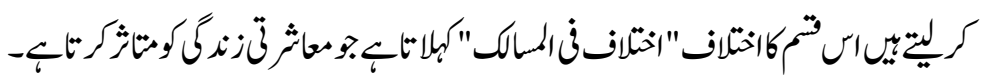

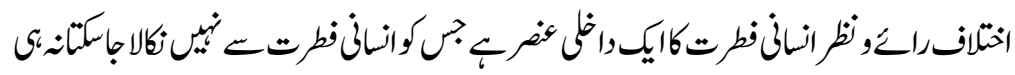

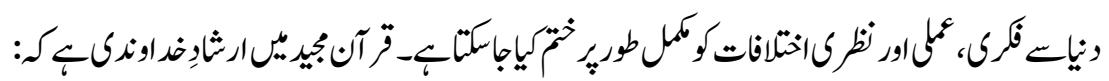

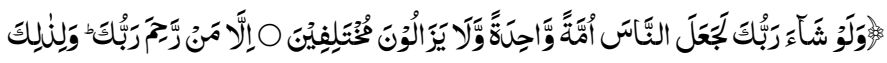

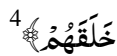

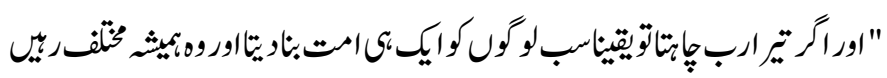

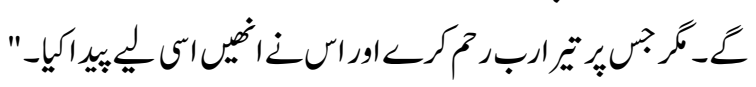

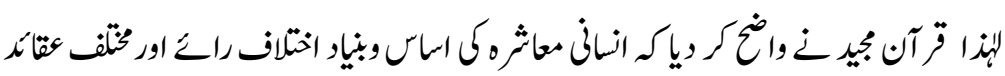

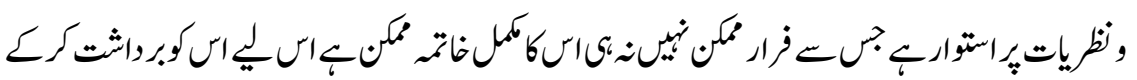

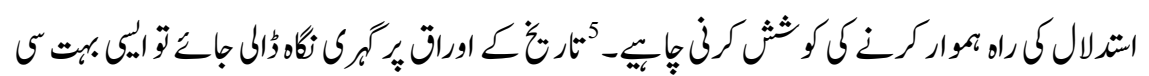

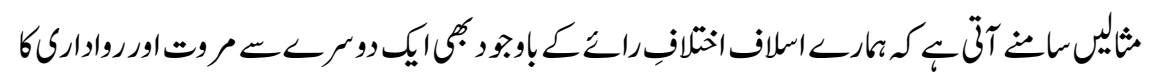

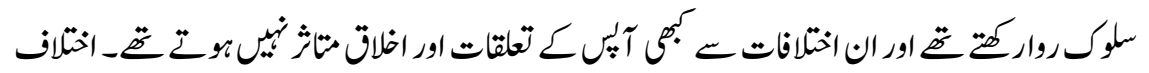

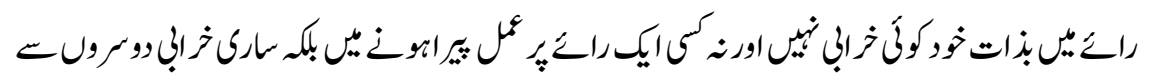

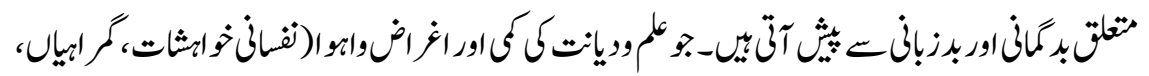

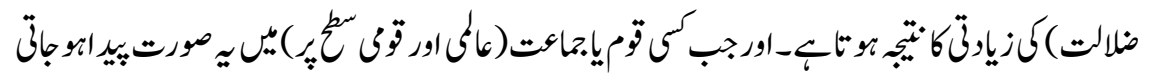

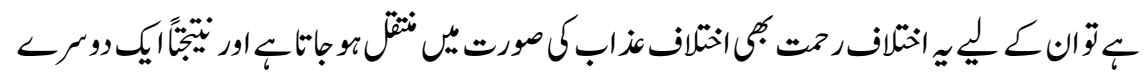

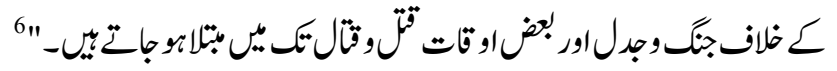




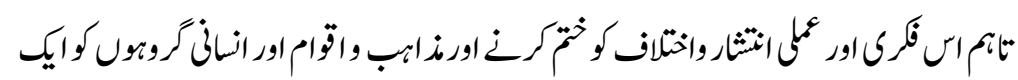

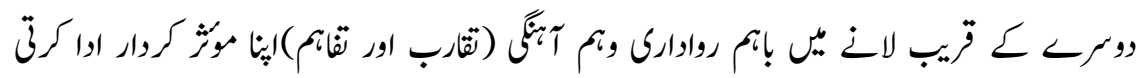

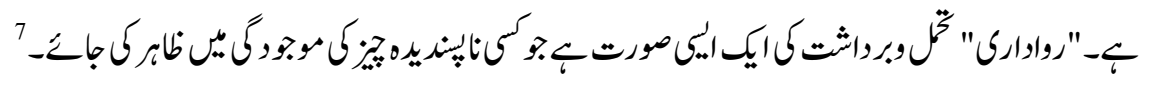

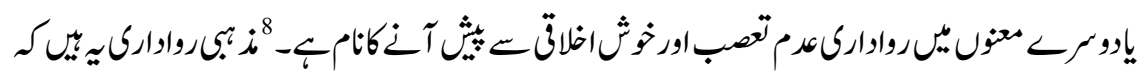

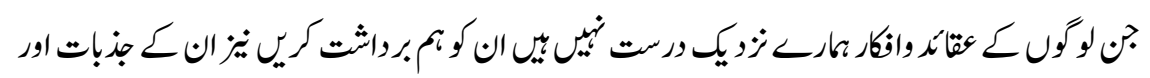

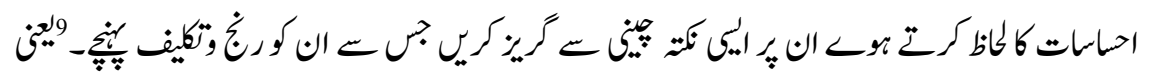

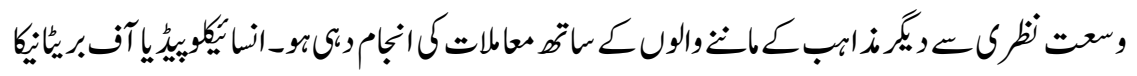
Encyclopedia of Britannica

"Intellectual and practical acknowledgement of the right of others to live in accordance with religious beliefs that are not accepted as one's own." 10

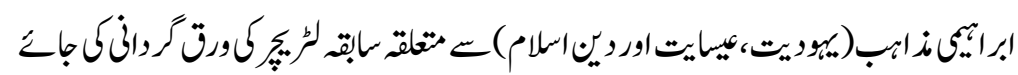

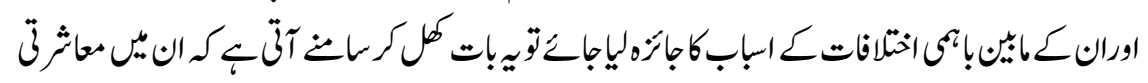

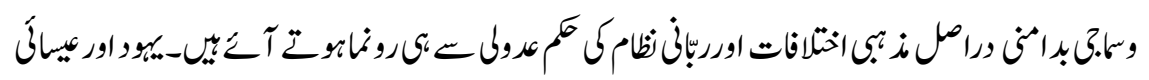

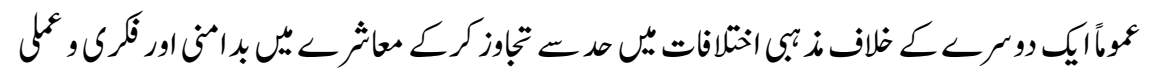

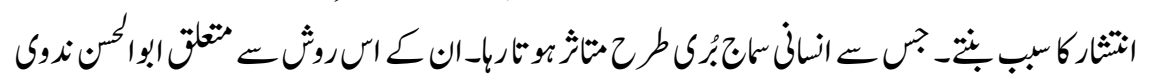

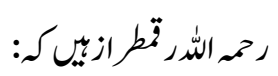

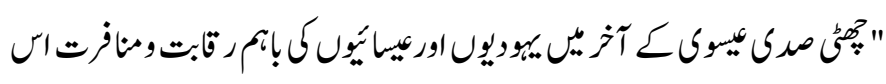

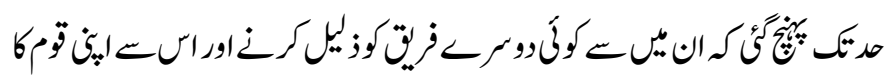

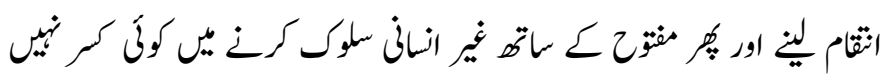

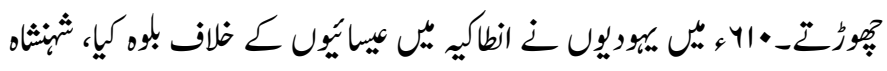

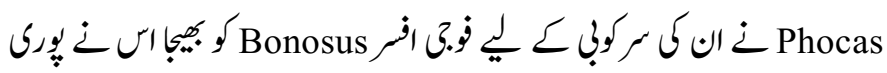

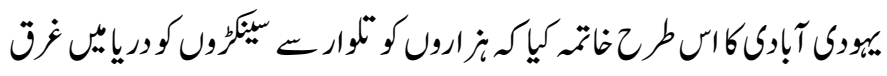

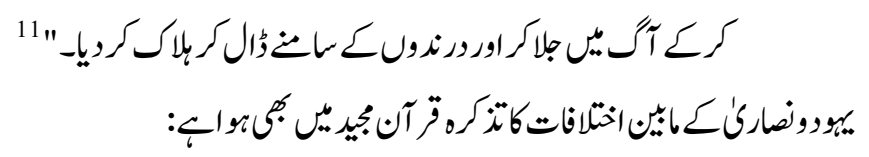




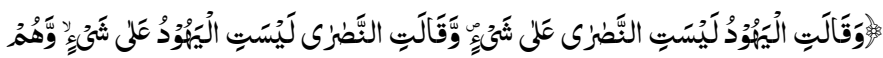

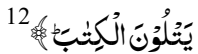

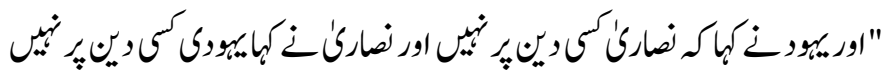

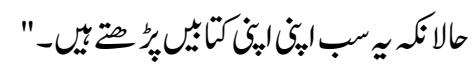

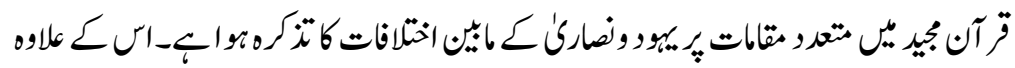

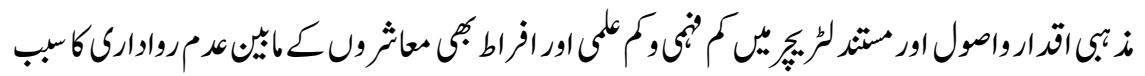

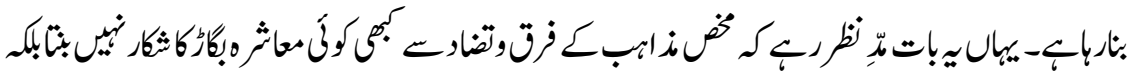

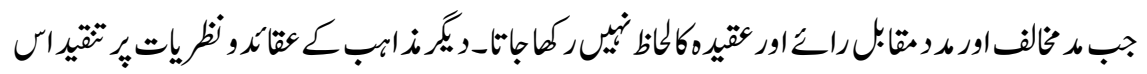

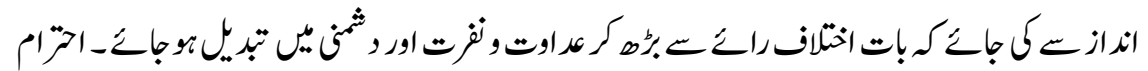

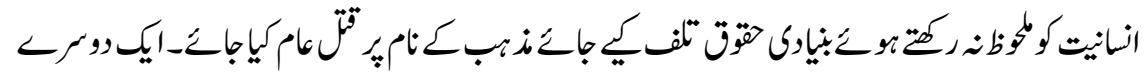

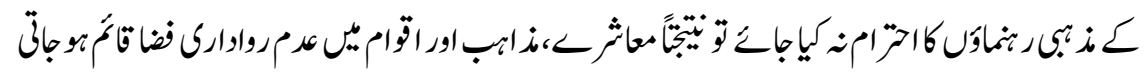

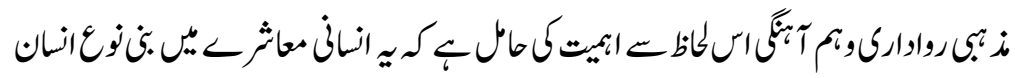

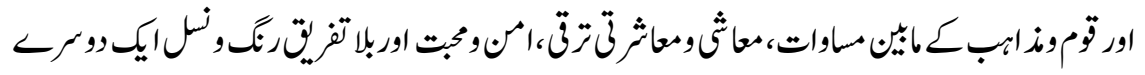

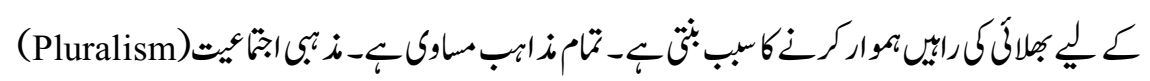

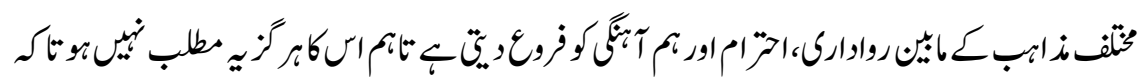

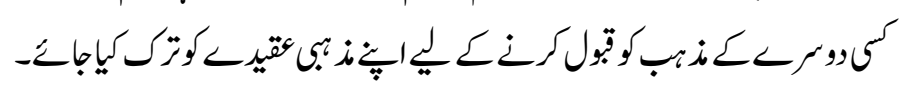

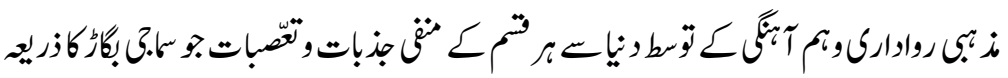

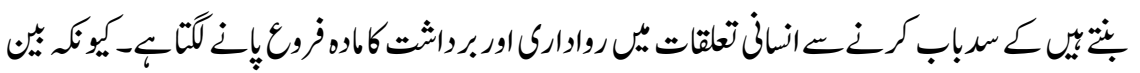

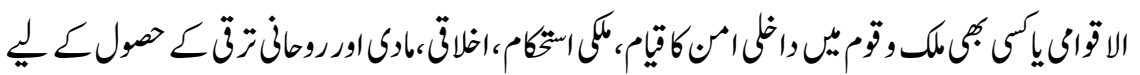

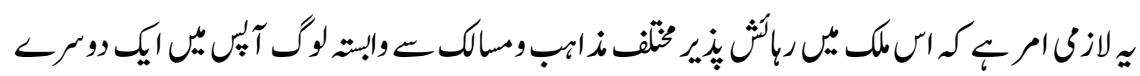

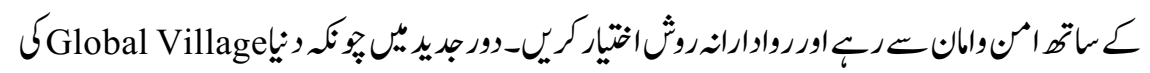

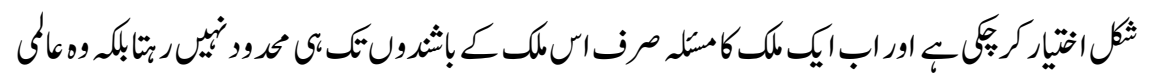

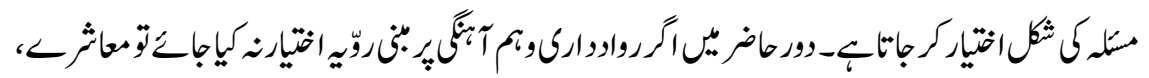




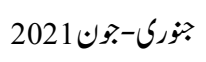

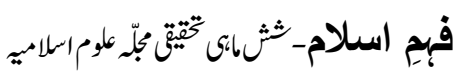

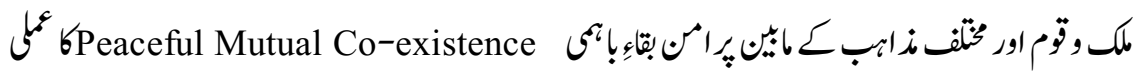

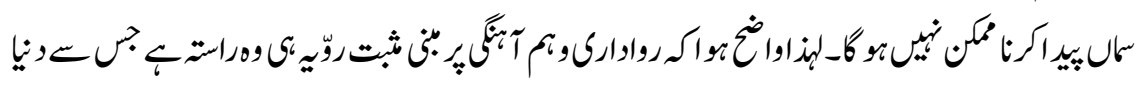

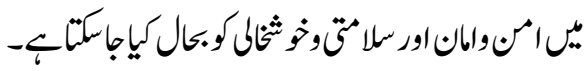

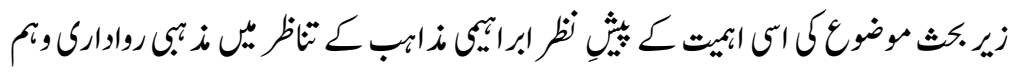

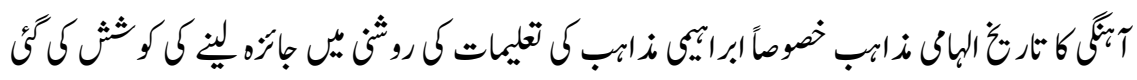

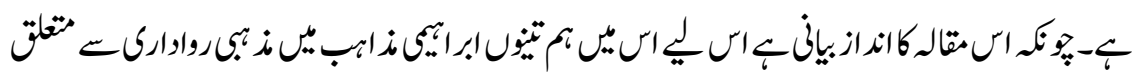

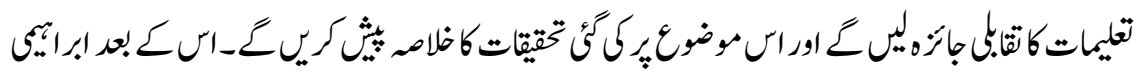

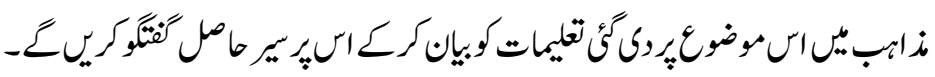

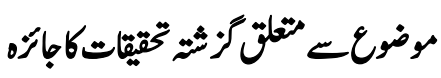

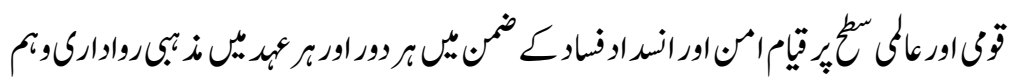

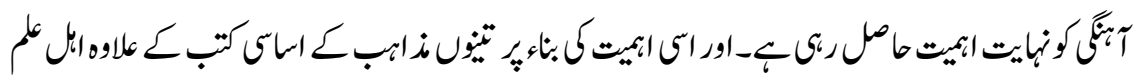

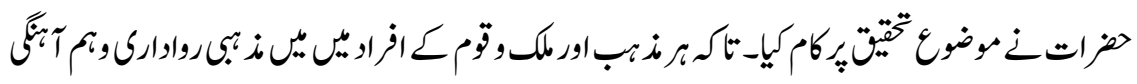

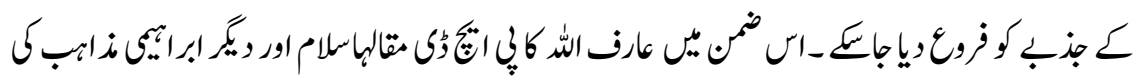

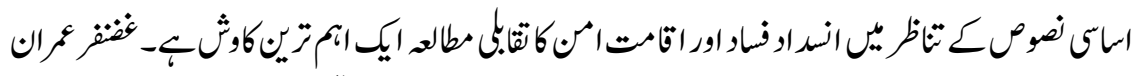

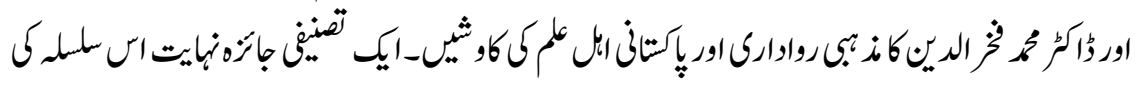

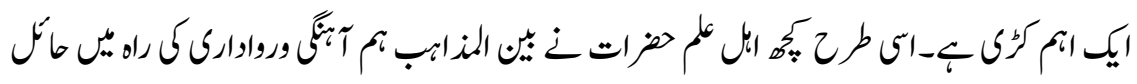

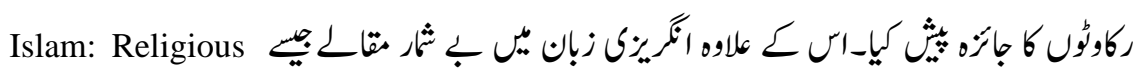

Understanding the Concept of ور Pluralism, and Modern Interpretations

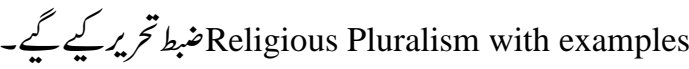

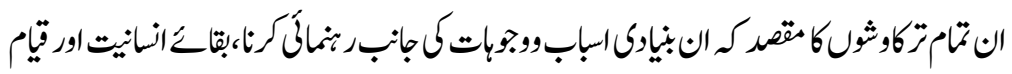

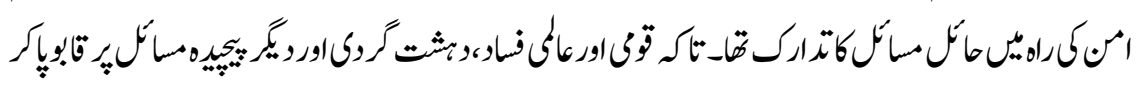

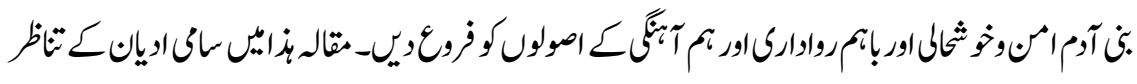

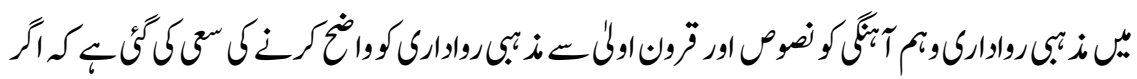

28 


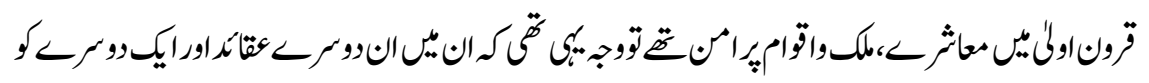

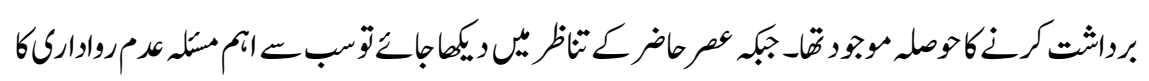

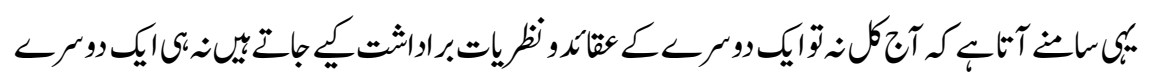

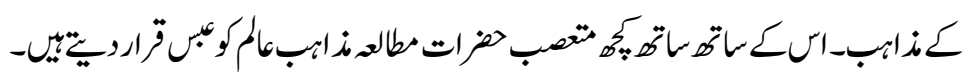

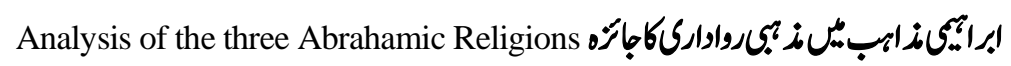

\section{ميورنذاببينرواراركى تليماتكاباءه}

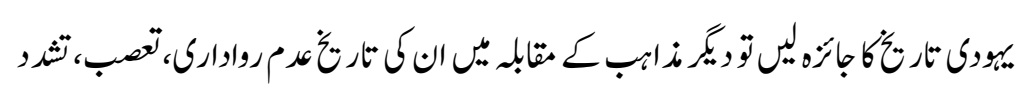

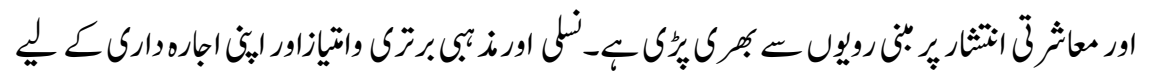

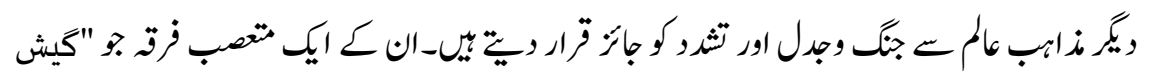

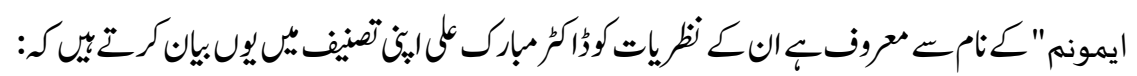

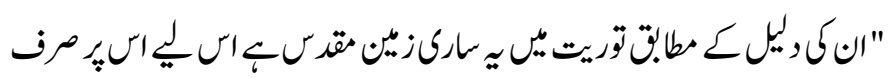

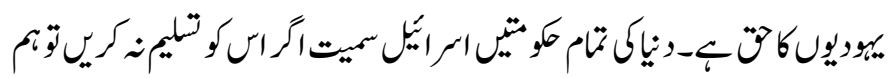

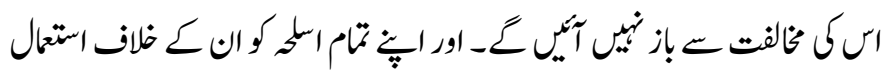

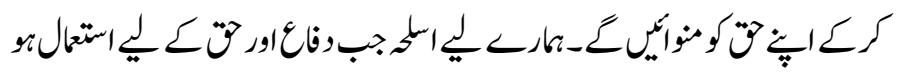

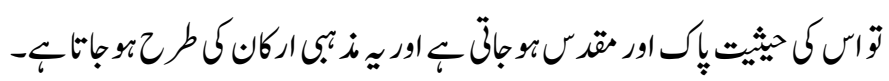

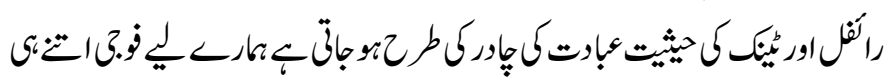

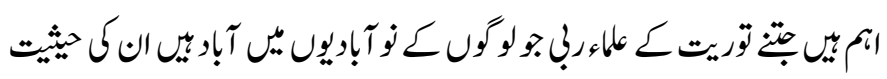

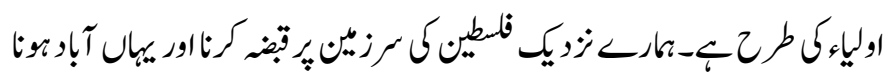

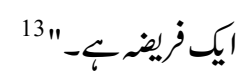

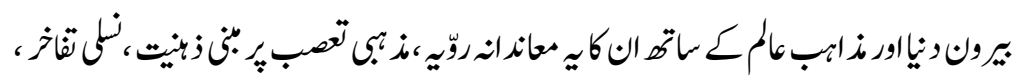

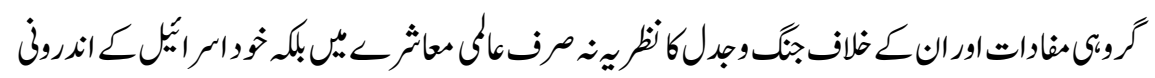

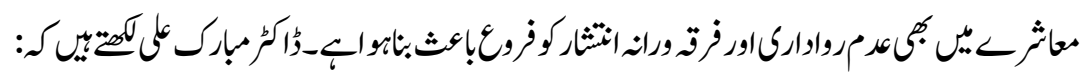




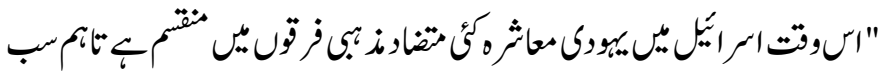

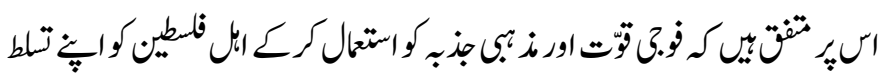

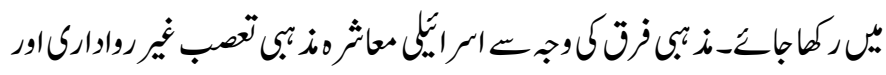

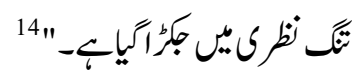

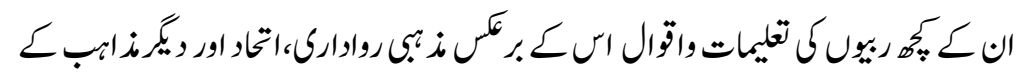

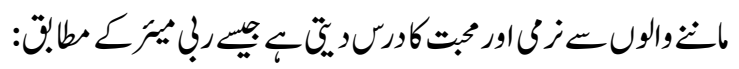

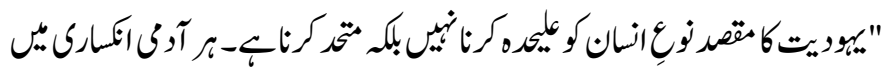

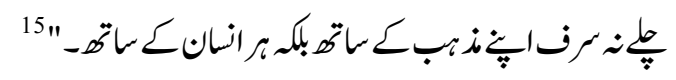

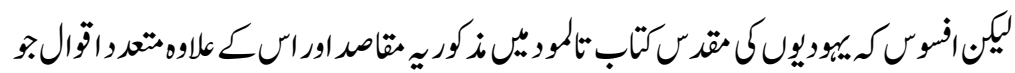

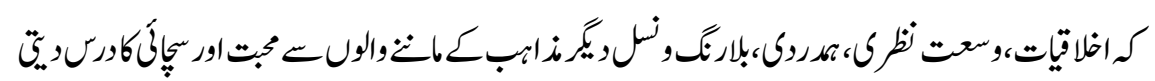

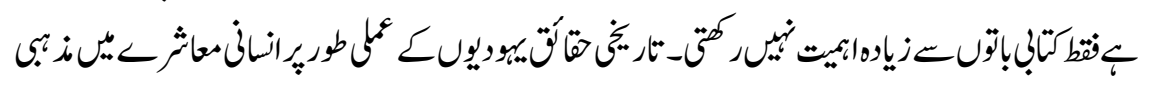

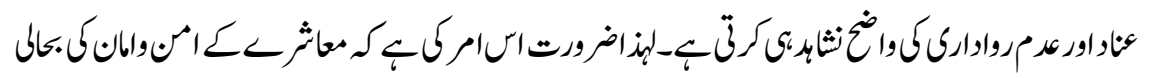

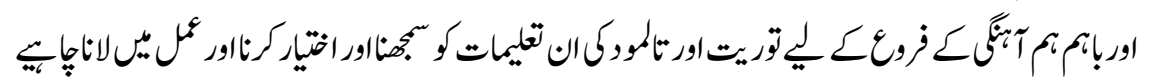

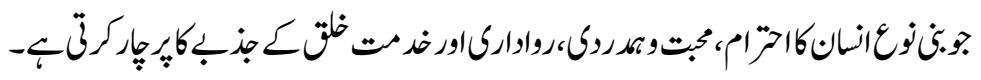

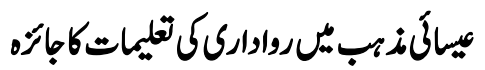

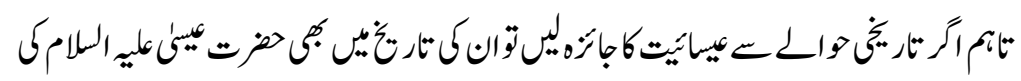

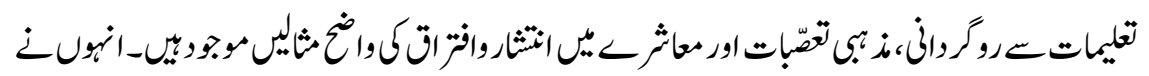

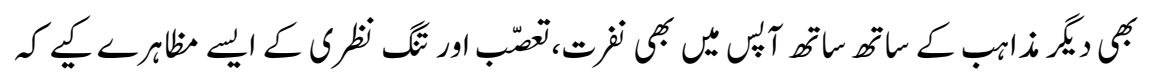

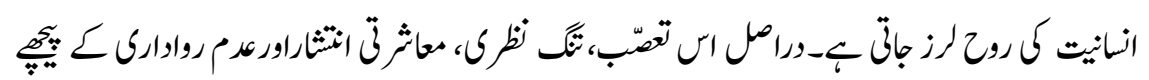

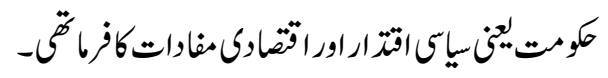

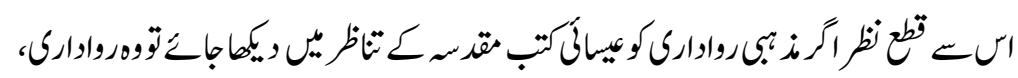

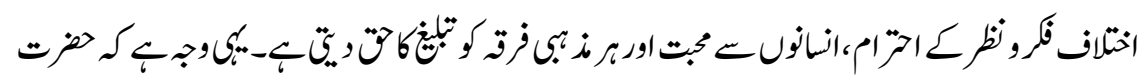

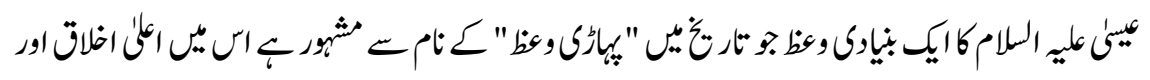




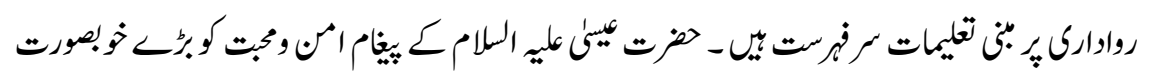

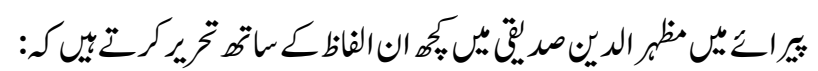

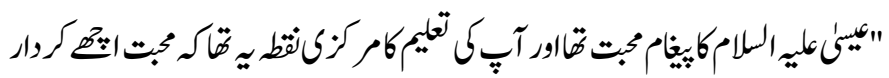

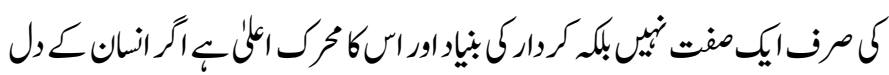

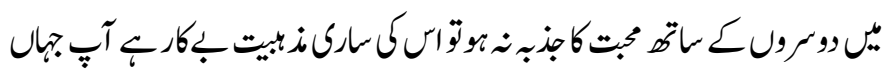

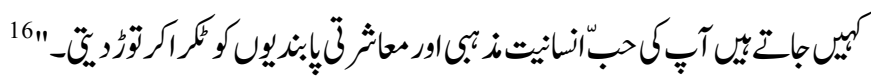

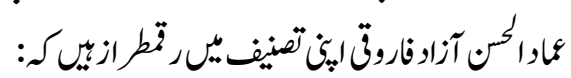

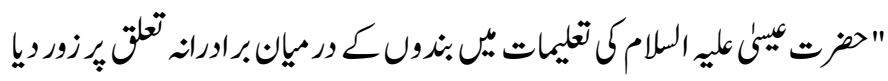

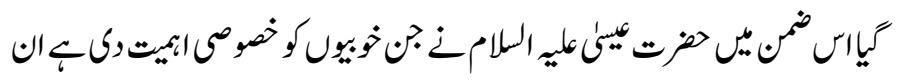

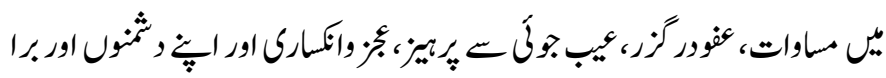

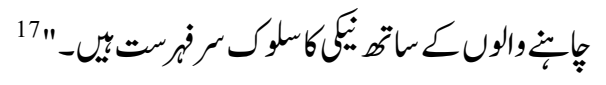

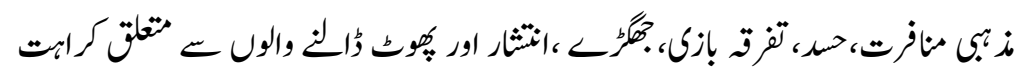

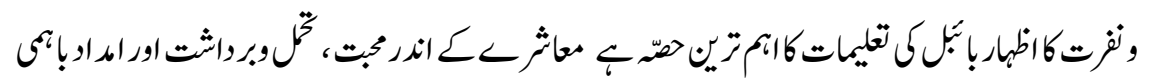

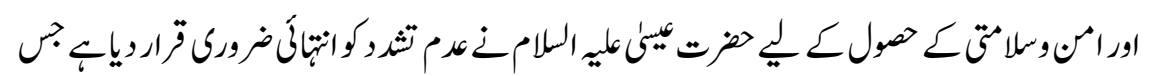

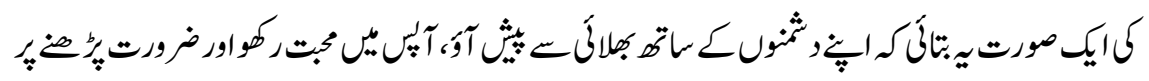

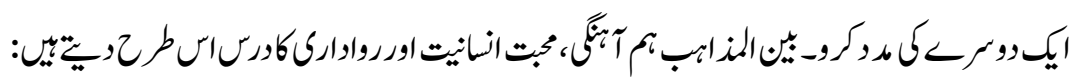

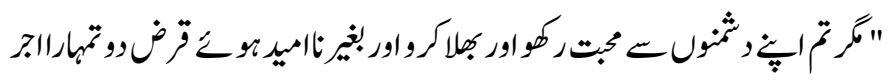

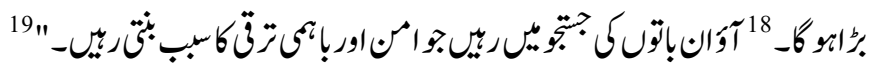

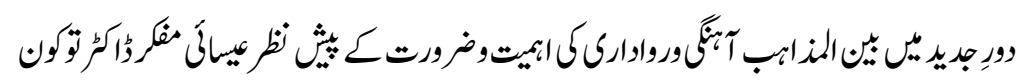

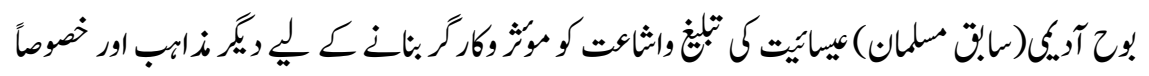

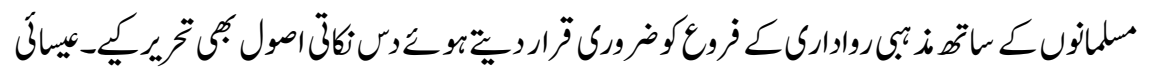

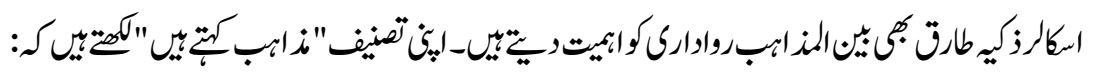




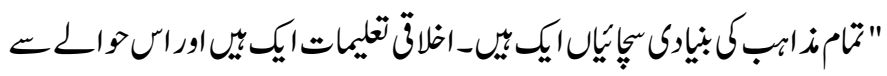

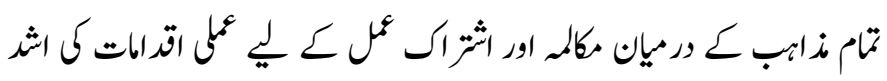

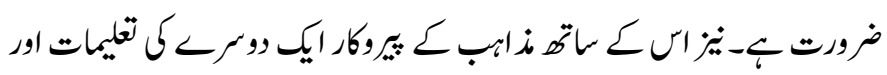

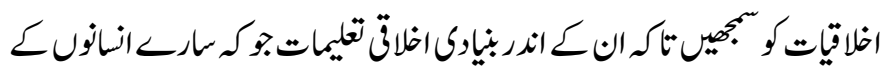

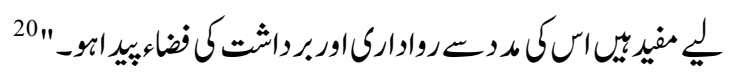

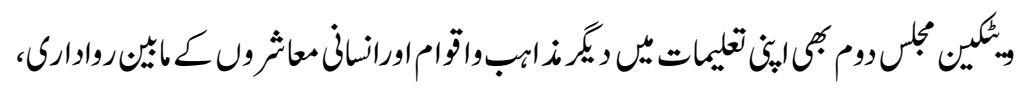

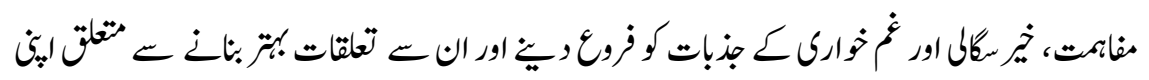

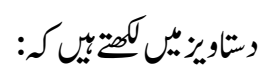

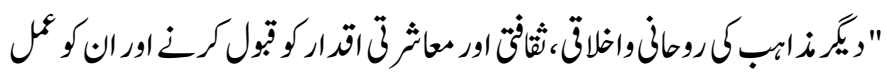

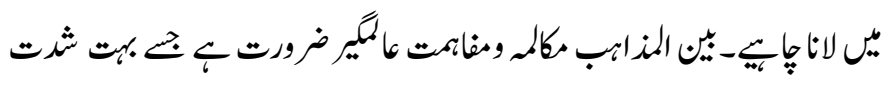

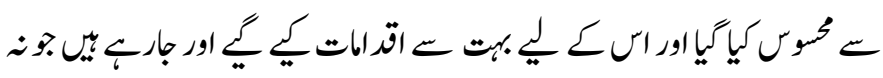

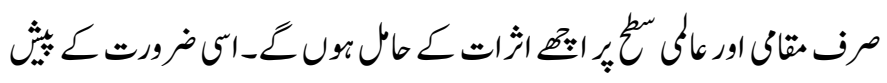

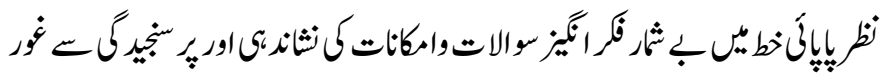

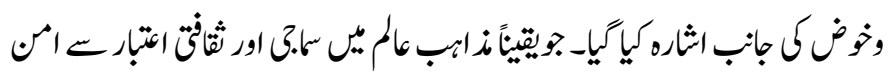

$$
\text { ك قيام اور مقصد ك لي منير }
$$

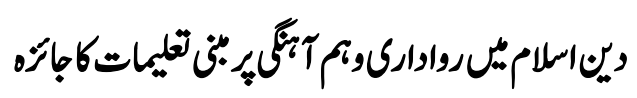

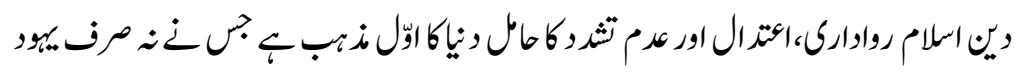

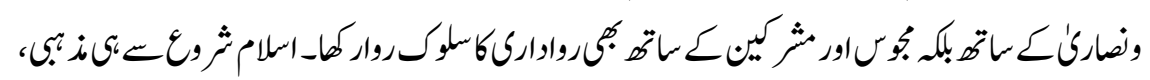

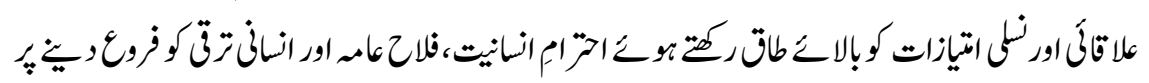

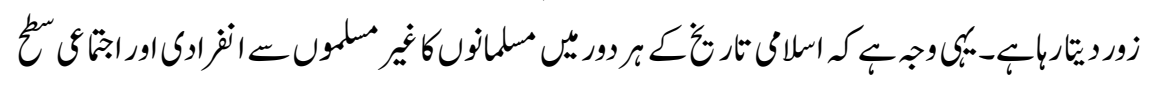

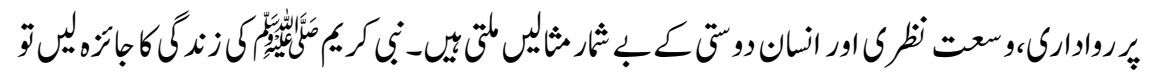

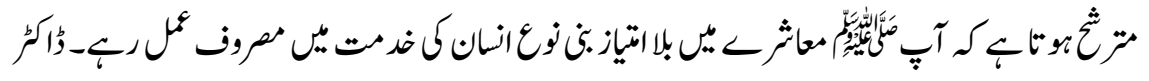

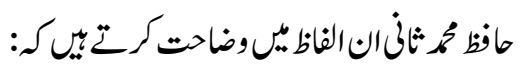




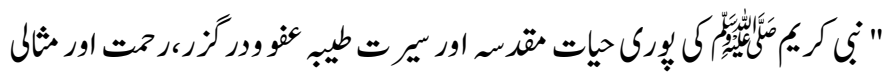

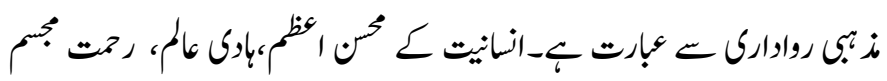

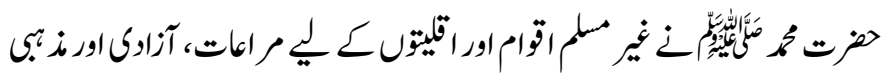

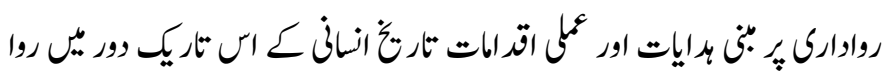

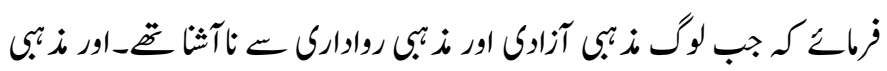

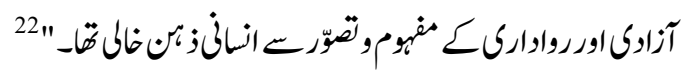

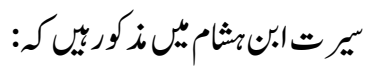

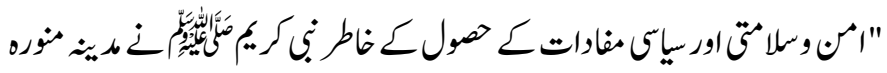

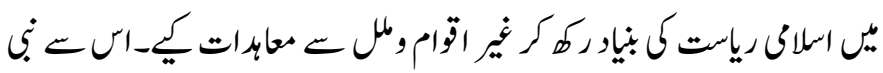

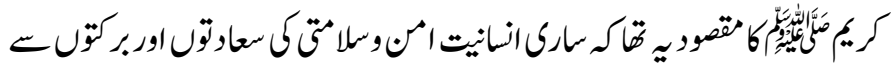

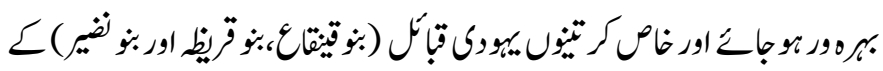

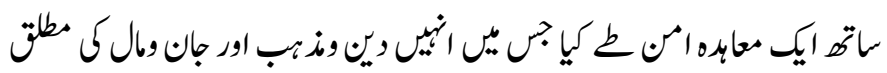
أزاوكوكئُ-

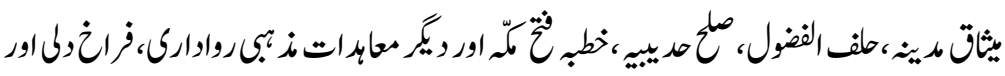

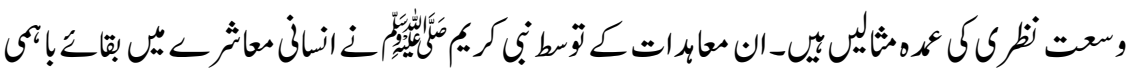

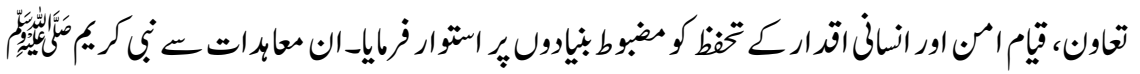

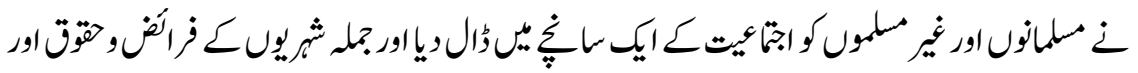

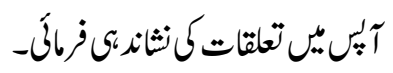

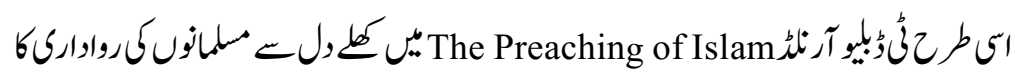

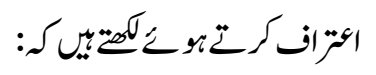

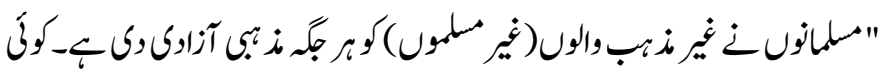

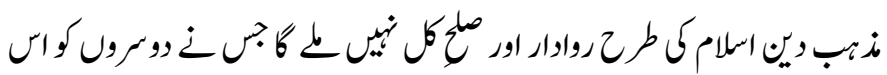




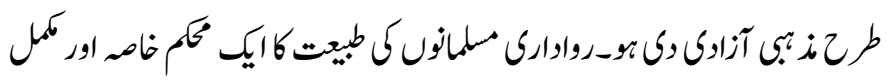

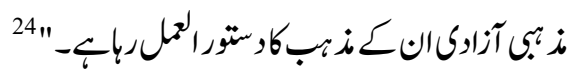

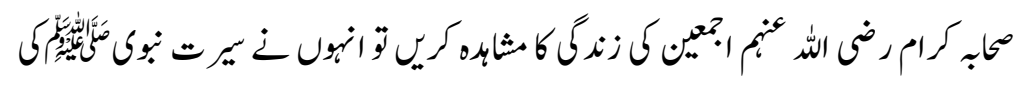

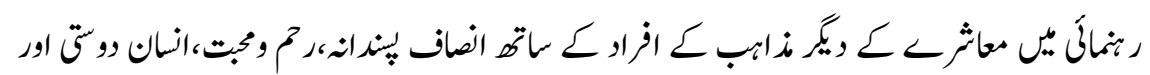

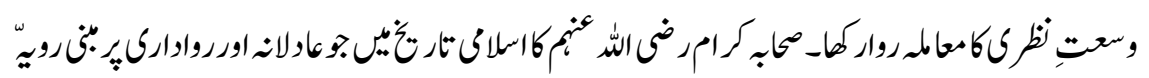

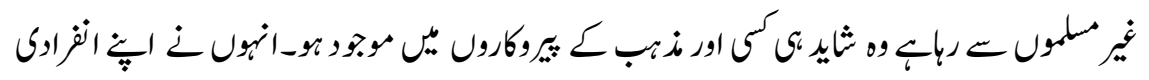

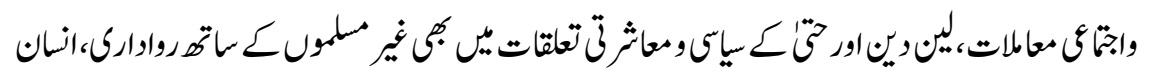

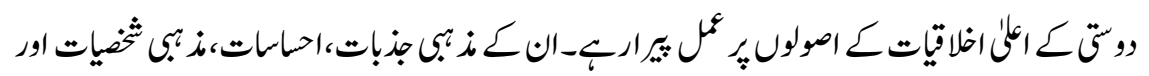

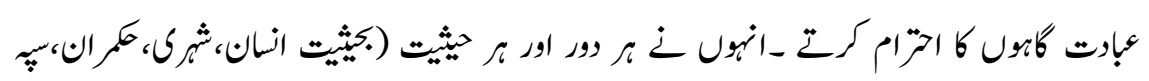

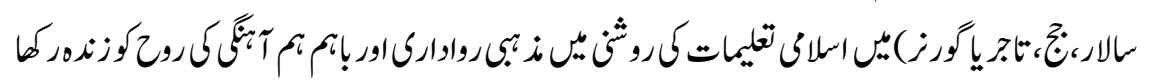

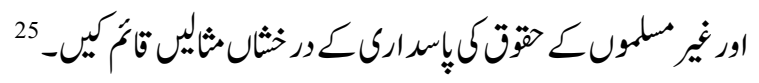

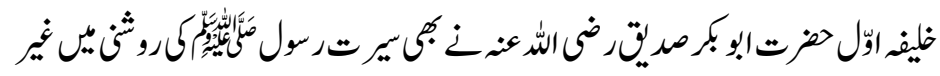

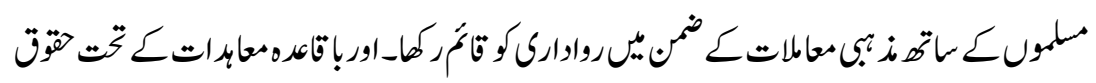

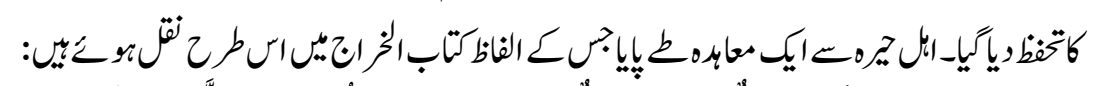

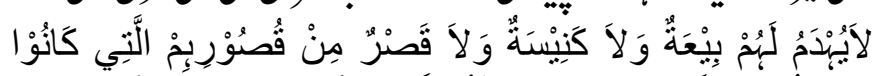

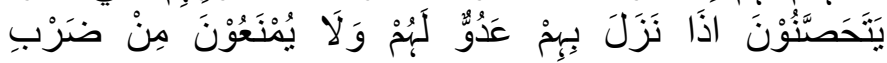

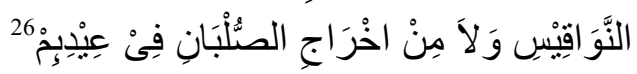

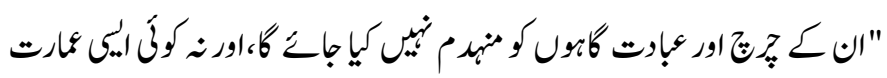

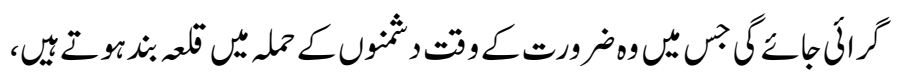

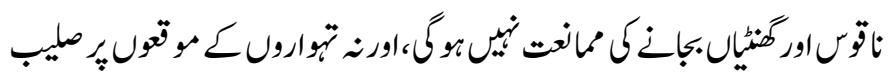

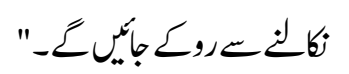

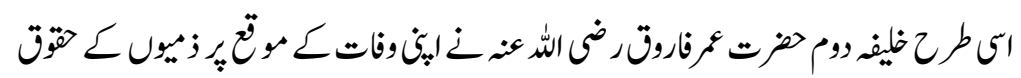
ع متحلق ليولوصيت فراكَ: 


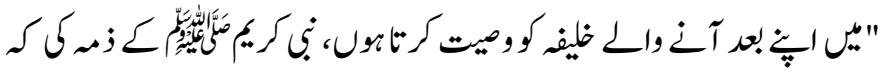

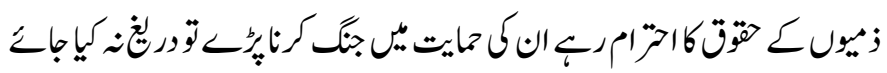

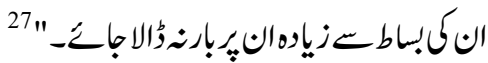

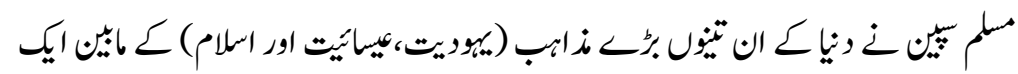

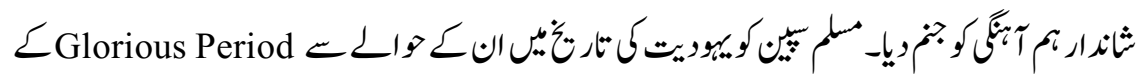

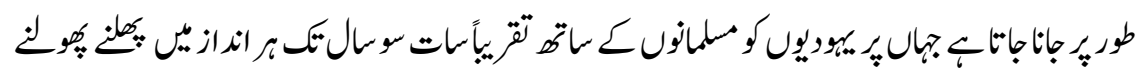

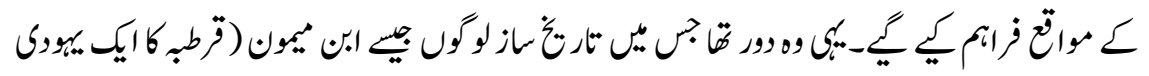

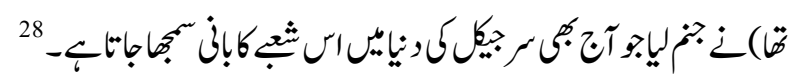

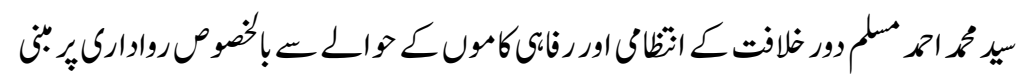

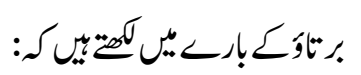

"Jewish intellectuals seeking a historical precedent for a more tolerant attitude towards Jews hit upon a time and place that met this criterion medieval Muslim Spain there they believed Jews had achieved a remarkable level of toleration, political achievement a cultural integration. ${ }^{29 "}$

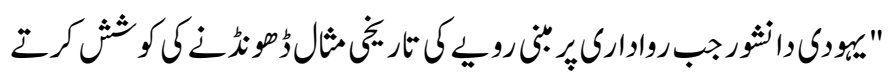

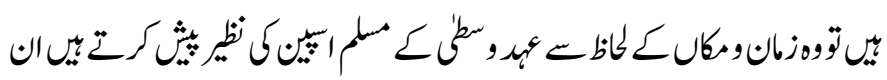

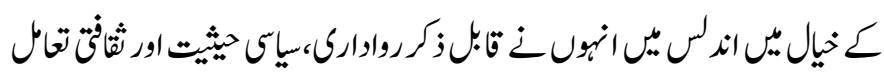

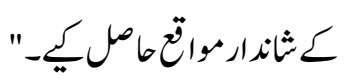

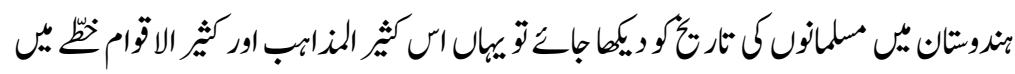

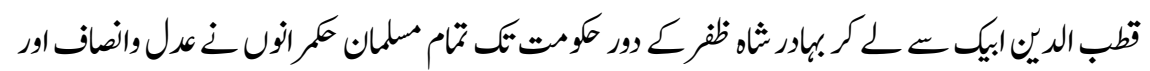

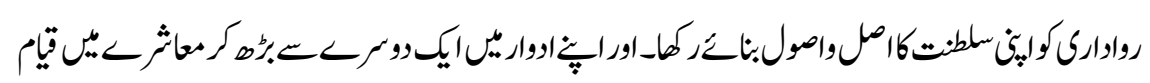

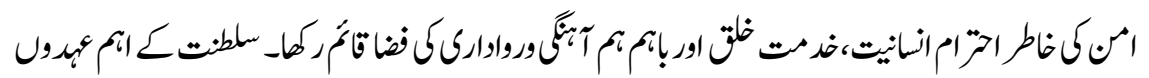

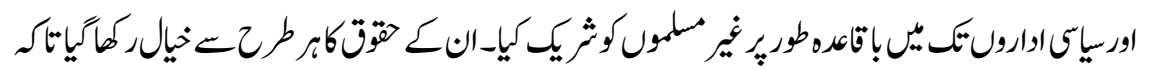

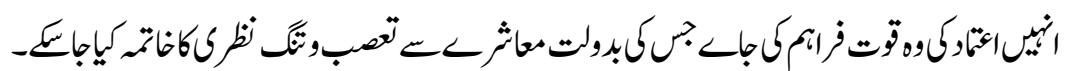




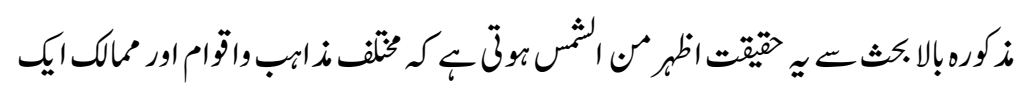

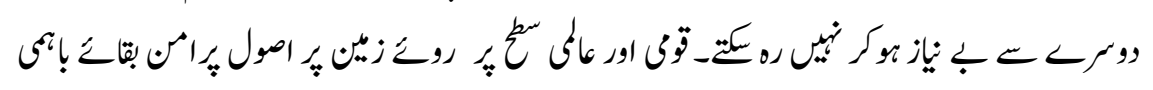
Peaceful Mutual Co- existence

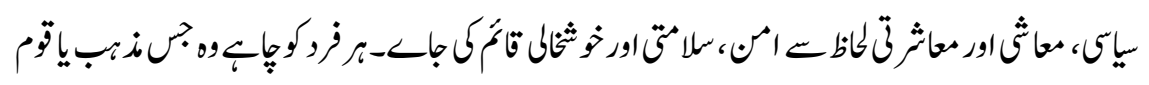

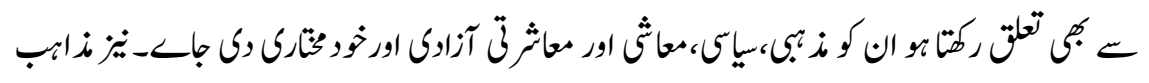

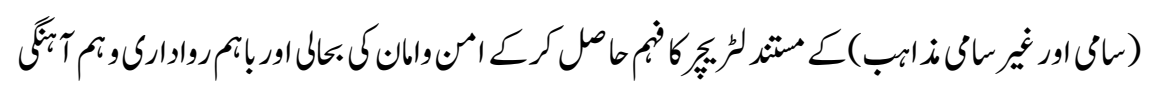

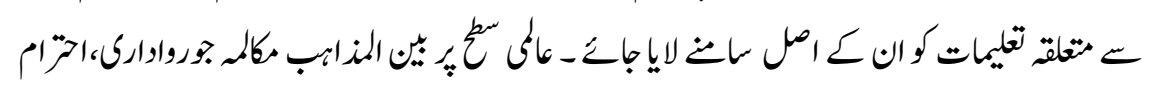

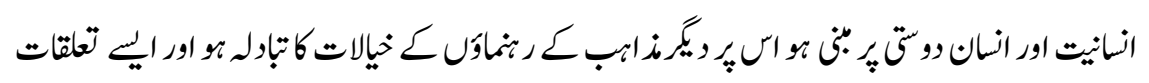

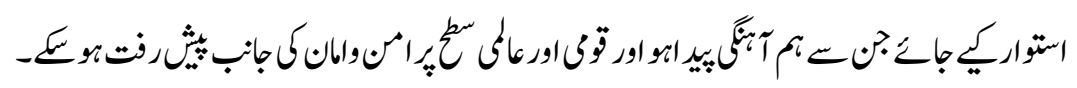

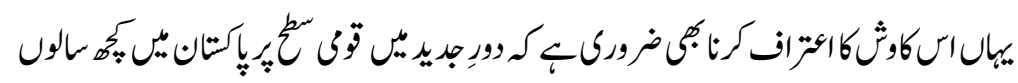

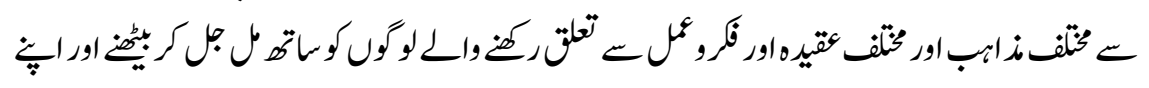

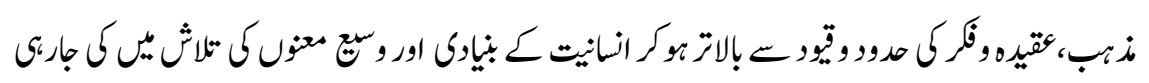

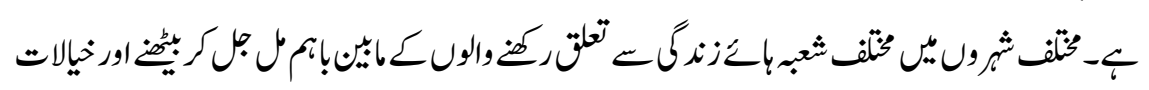

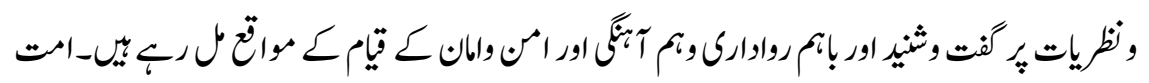

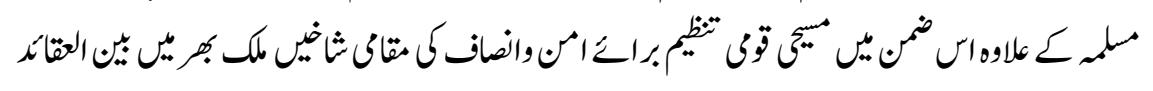

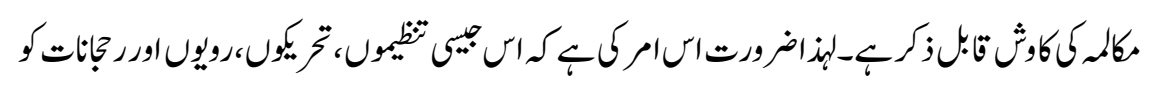

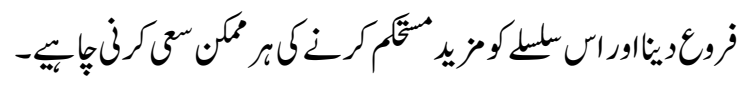

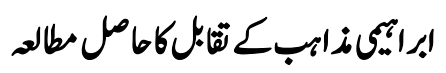

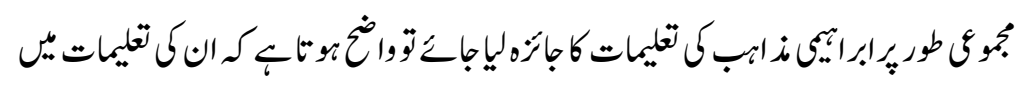

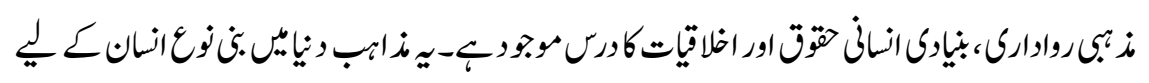

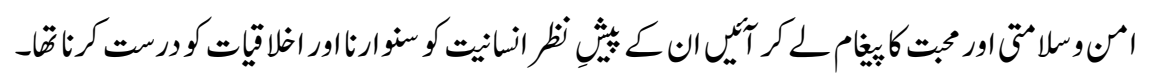

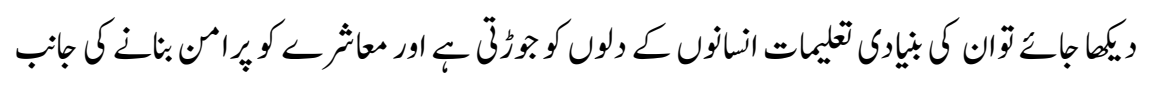

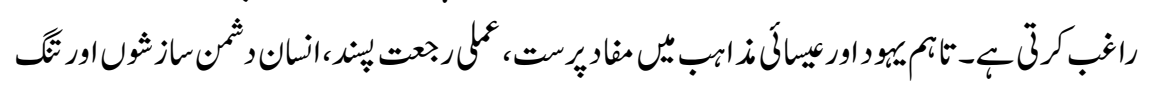




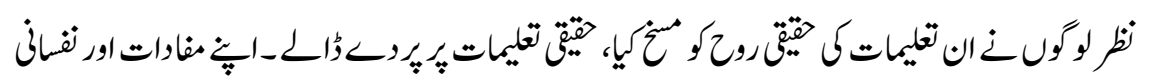

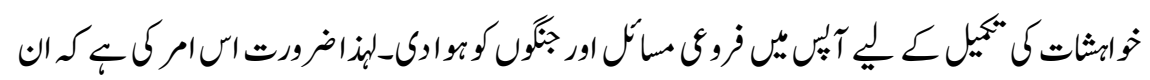

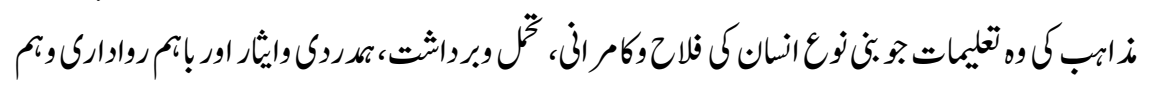

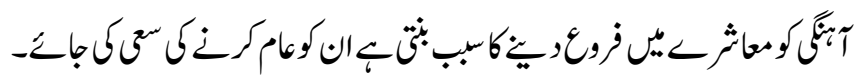

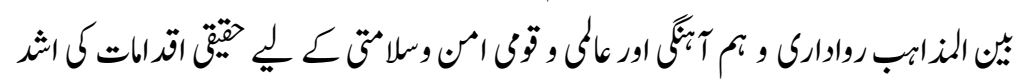

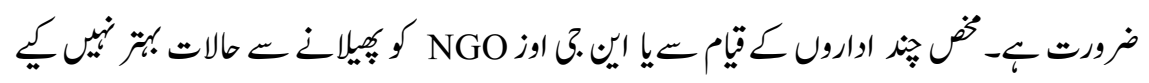

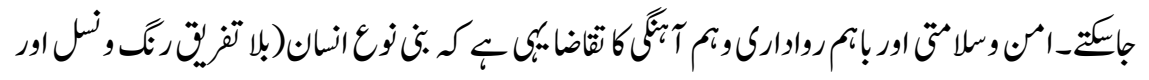

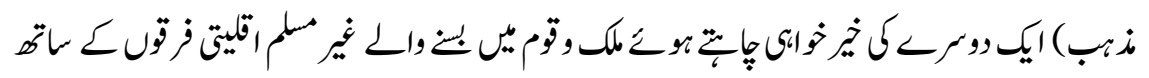

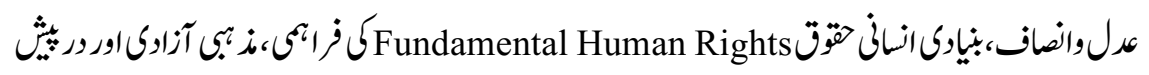

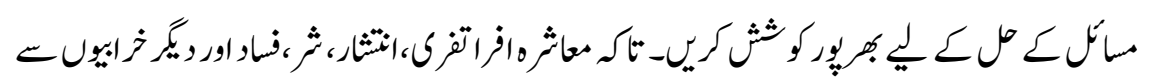

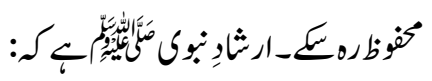

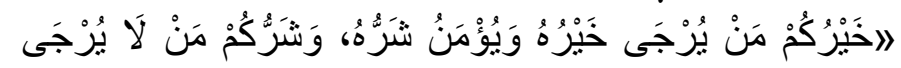

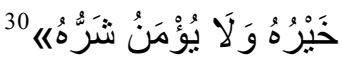

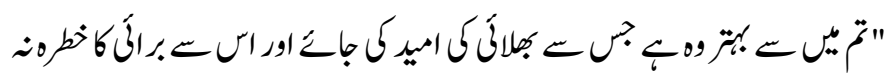

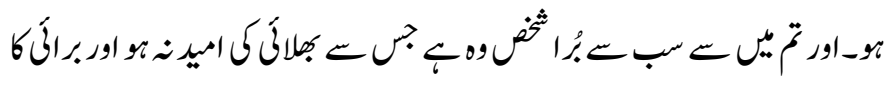

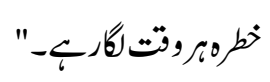

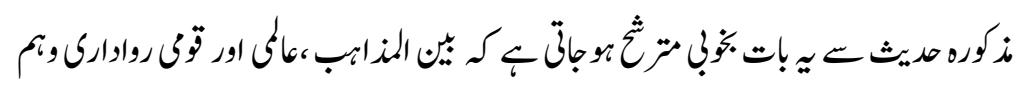

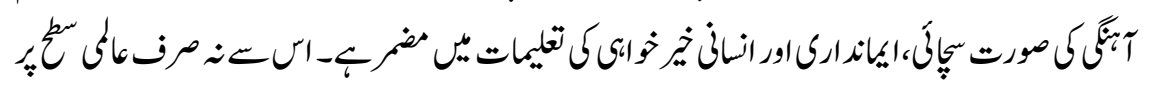

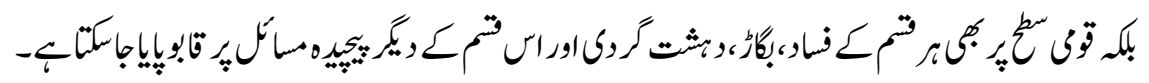

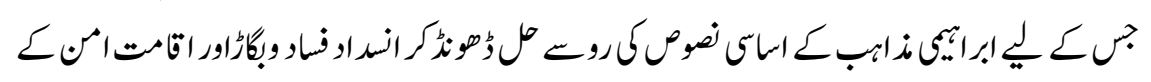

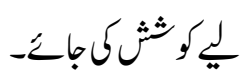

تخجيزوسنارثات 


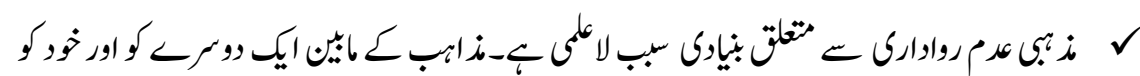

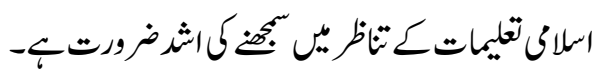

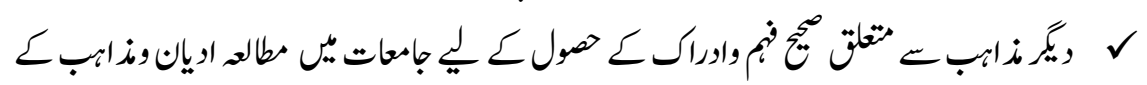

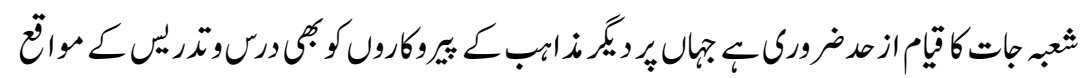

$$
\text { - }
$$

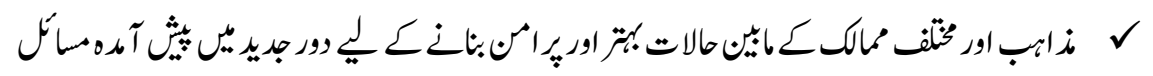

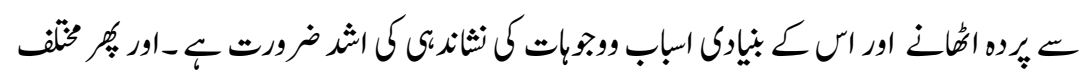

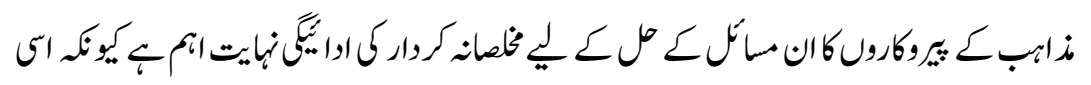

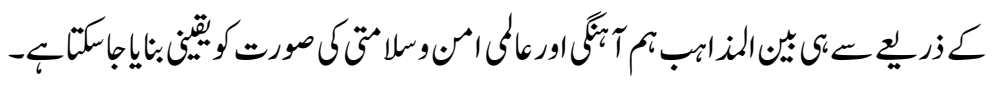

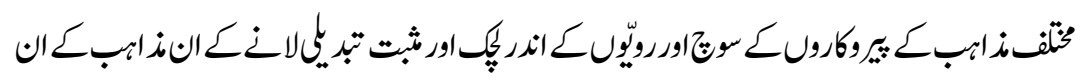

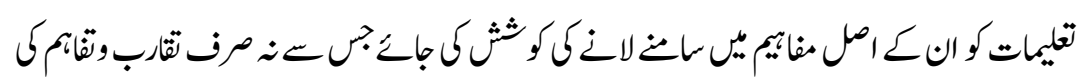

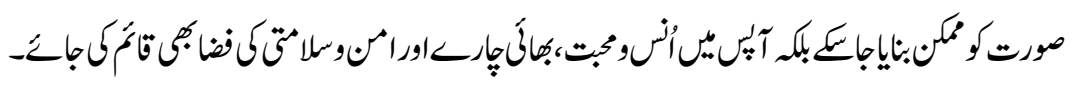

\section{مصاوروالمرانع}

Al Qur'an, Surah-tul-Isra:84.

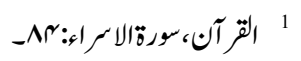

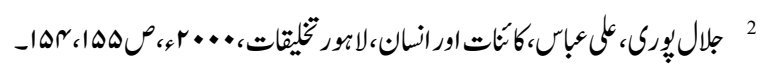
Jalal Pori, Ali abbas, Kainat or Insan, Lahore Takhliqaat, 2000,P\# 154,155.

$$
3
$$

Mubarak Ali (Dr.), Tarikh Kiya kehti hai, Fiction House, Lahore,1998,P\#90.

Al Qur'an, Surah-tul-Hood:118,119.

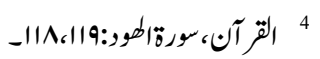

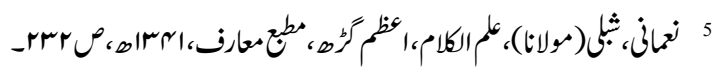

Numani, Shibli (Maulana), Ilmul Kalam, Aazm Ghar, Matba Ma'rif, 1342 Hijri, P\#232.

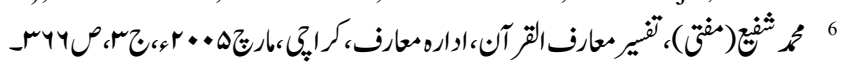

Muhammad Shafi (Mufti), Tafseer marif -ul-Qur'an, Idara marif, Karachi, March 2005, Vol 3, P\#366.

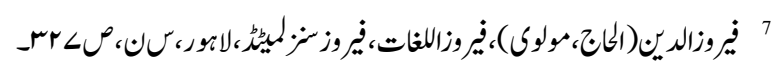

Ferozuddin (Alhaaj, Molvi), Feroz-ul-Lugaat, Feroz sons Limited, Lahore, P\#327. 


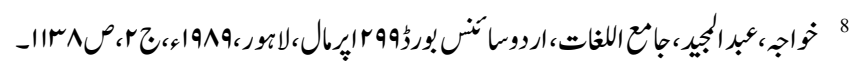

Khuwaja, Abdulmajeed, Jamiul Lugaat, Urdu science board 299 upprmal, Lahore,1989,Vol 2,P\# 1138.

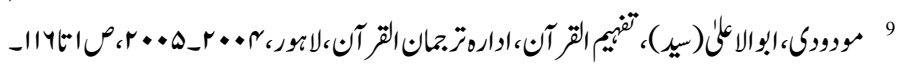

Mododi, Abul ala(Syed), Tafheemul Qur'an, Idara tarjuman ul Qur'an, Lahore, 20042005, P\# 1-116.

10 Johannes P, Becchio Bruno, Encyclopedia of world Religions, Concord publishing foreign media books,2006, Section 31.

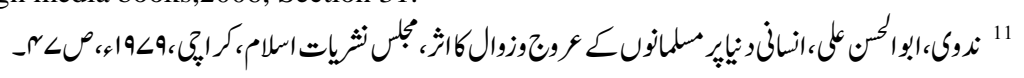

Nadvi, Abulhasan Ali, Insani Duniya Par Musalmano ky Urooj o Zawaal ka Asar, Majlis Nashriyat Islam, Karachi, 1979,P\#47.

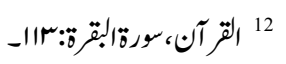

Al Qur'an, Surah-tul-Baqara:113.

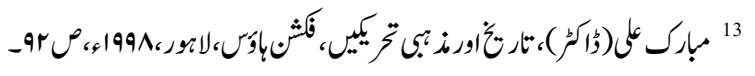

Mubarak Ali(Dr.), Tarikh or Mazhabi Tehrekain, Fiction House, Lahore, 1998, P\#92.

$$
14
$$

Ibid.

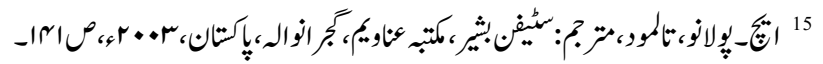

H-Polanco, Talmud, Mutarajjim: Stefon Bashir, Maktaba anaveem, Gujranwala, Pakistan, 2003,P\# 141.

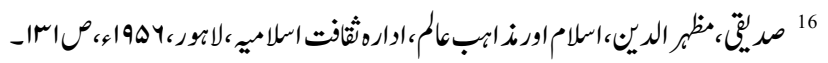

Siddiqi, Mazhar ud deen, Islam or Mazahib e Aalam, Idara saqafat e Islamia, Lahore, 1956, P\# 131.

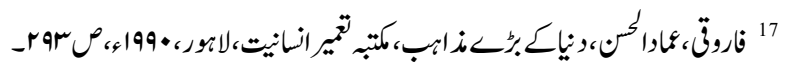

Farooqi, Imaad ul Hasan, Duniya k bary Mazahib, Maktaba Tameer e Insaniat, Lahore, 1990,P \# 293.

Kiataab e Muqaddas, Injeel Loqa, 6/35.

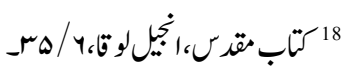

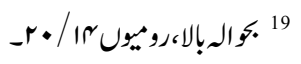

Ibid, Romeo, 14/20.

$$
20
$$

Zakia Tariq, Mazahib Kehty Hai, Maktaba anaveem, Gujranwala, 2003,P\# 92.

$$
\text { 21 }
$$

Ibid. 
Muhammad Sani(Hafiz, Dr.), Rasool Akram蹲 or Rawadari, Fazli sons, Lahore, 1988,P\# 50.

23

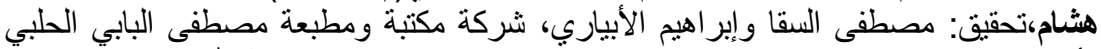

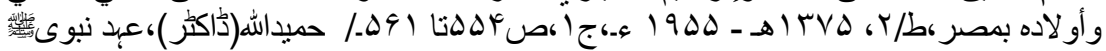

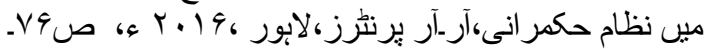

Ibn Hashaam, Abdul Malik bin Hashaam bin Ayub Alhumairi(213 Hijri), Al-seera tul Nabvia Le Ibn Hashaam, Tehqeeq : Mustafa Alsaqa wa Ibrahim Al-abyari, Sharika Maktaba wa Matba Mustafa Albabi Alhibli wa aulaada, bemisar, Edition 2, 1375 Hijri1955,Vol 1,P\#554 to 561./ Hamidullah (Dr.), Ahd Nabavi数 main Nizam e Hukumrani, R-R Printers, Lahore, 2016,P\# 76.

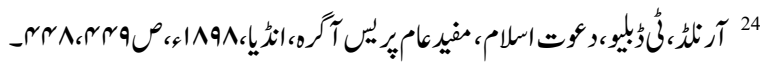

Arnild, T.W, Dawat e Islam, Mufeed Press Agrah, India, 1898,P \# 448,449.

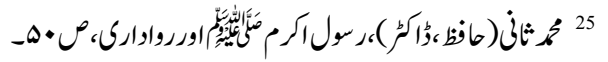

Muhammad Sani(Hafiz, Dr.), Rasool Akram or Rawadari, P\# 50.

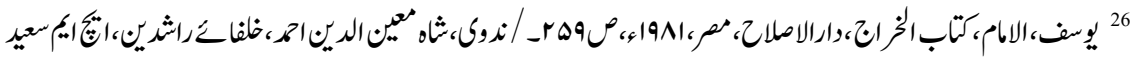

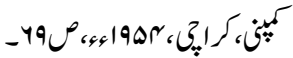

Yousuf, Al-imam, Kitab ul Khiraaj, Dar ul Islah, Misar, 1981,P\# 259.

$$
27
$$

Ibid.

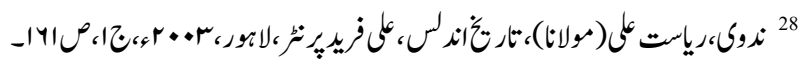

Nadvi, Riyasat Ali(Moulana), Tarikh Andolas, Ali Fareed Printer, Lahore, 2003,Vol 1,P\# 161.

29 Ahmad, Muhammad (Syed), History of Spain, Pakistan R.R Printers, Lahore,2010, P\#574.

30

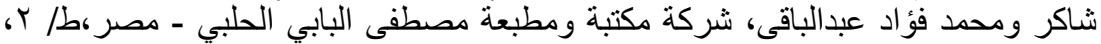

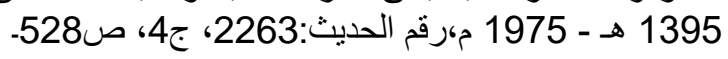

Al-Termizi, Abu Esa, Muhammad bin Esa (279 Hijri), Tehqeeq wa Taleeq: Ahmad Shakir wa Muhammad Fawad Abdul baqi, Sharika Maktaba wa matba Mustafa Albabi Alhalbi-Missr, Edition 2, 1395 Hijri-1975, Raqmul Hadees: 2263, Vol 4,P\# 528. 